\title{
Generation of the Tsunami in Compressible Water (Part I)
}

\author{
By
}

\section{H. MiYoshI}

(Geophysical Institute, Tokyo University)

\begin{abstract}
In this paper is given a theoretical investigation of a disturbance which may occur on the surface of the sea as the effect of an elevation or a depression of the bottom. The water is thought to be compressible and the sea floor is assumed to be consisted of a rigid substance. It is shown that the sea surface oscillates up and down as the result of waves of expansion occuring in the water by the dislocation of the bottom. The period of oscillation depends upon the depth of the water and is of the same order as that of usual swell.
\end{abstract}

\section{§1. Introduction}

The average depth of the sea floor, at which tsunamis occur has been considered to be several thousand meters." When the depth is large, the effect of the compressibility of the sea water is not negligible. $t$ Actually, when a very wide area of sea floor elevates (depresses), the water mass is supposed to be piled up on the sea surface, presenting a same form as that of the deformed part of the bottom. But this is the case in which the sea water is incompres-

* A catalog listing 270 tsunamis recorded all over the world during the period 479 B. C.1946 A. D. was published by N.H. Heck (1947). Instead of his comprehensive list, we will only place here a small map showing the origins of the seven tsunamis which attacked the northern parts of Japan (after A. Imamura, 1934). The average depth of their origins is about three thousand meters.

$\dagger$ According to seismologists, the velocity of the bottom deformation is considered to be as rapid, from analogies, as that of the phenomenon of the fault on the land.

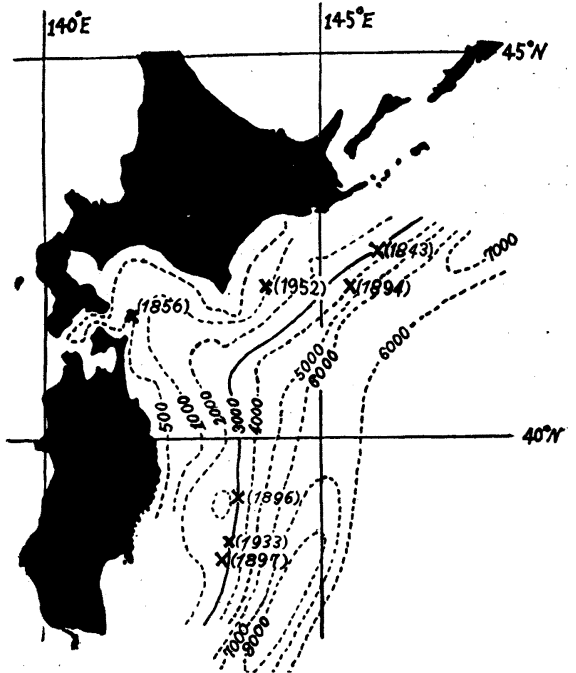

Fig. 1 
sible. When the sea water is compressible, there will always occur waves of expansion in the water. Consequently, the surface elevates or depresses as the result of the vertical accompanying oscillation. This oscillation closely resembles that of a mass hanged by a spring, in which the energy of oscillation is much smaller than it appears, and has not an effect on the earthquake. But in this case, the spring and the mass are identical-the water. The period of this oscillation is the time required for the sound wave to travel between bottom and surface four times, and is the order of that of the usual swell, in the water of above-mentioned depth. And this oscillation is magnified or ceases at the origin after the bottom deformation, depending upon the time interval during which the latter was performed. This motion of shrinking and stretching of the water column is supposed to be of considerably large scale. Because the horizontal and vertical dimensions of the bottom deformation are several hundred kilometers and several meters, judged from the refraction diagram and the total energy of the tsunami caught along the coast, respectively. And the disturbances, which are caused by this oscillation and expected to form the forerunners of a tsunami, are not caught at the coast but mostly absorbed into the bottom crust at the origin and near the coast. How much part does this oscillation occupy the so-called sea-shock and the $T$-phase, we cannot say with confidence. Repeatedly saying, the period of this oscillation is the order of that of the usual swell on the surface of deep sea and grows quicker when it comes to the shallow sea.

The appearance of this oscillation will be made complex, in addition to the irregularity of the bottom configuration and the wavy surface, for the following three reasons.

1. The non-uniformity of the sound velocity.

We need not take this seriously, for the deviations of the sound velocities in the sea water from the mean value are trivial.

2. Existence of the unconsolidated sediments.

M. Ewing and others (1950) reported from seismic refraction measurements in the Atlantic Ocean Basin, the existence of the unconsolidated sediments, the thickness and the surface slope of which are 4,500 feet and 20 minutes respectively. This substance will behave as a solid for the tsunami wave (judged from the slope of its surface, the occurence of such a one as the internal wave cannot be imagined), and as a liquid for the above-mentioned oscillation. For simplicity, we will neglect the effect of this sediments.

3. Penetration of the energy of this oscillation into the bottom. 
Energy ratio of the seismic waves reflected and refracted at a rock-water boundary was calculated in detail by K. Ergin (1952). According to his table, when the sound wave enters from water to the basic rock, its critical angles and the reflectional ratio are ten or so degrees ( $P$-wave), twenty or so degrees ( $S$-wave) and $60-70 \%$, respectively. So the wave which travels within the critical angle (approximately in a vertical direction) will almost disappear after travelling between bottom and surface several times. On the other hand, the wave which inclines outsides the critical angle, may reach the far distance zigzagging between bottom and surface. But it reaches the continental slope, its direction approaches the vertical line every zigzag, and will be absorbed into the crust.

The 3rd effect is the most serious, but we shall first neglect it. To describe the surface oscillation at the origin, we may imagine a cylinder long enough containing the water to lift and watch the motion of the surface. But, the waves of expansion will spread in all directions soon after. The latter procedure will be treated in Part II. We will treat these phenomena en bloc, as follows.

\section{§. General Analysis}

Let us assume the sea to have a uniform depth $H$, take the origin of the cylindrical coordinates at the undisturbed surface and represent the downwards coordinate by $U z$, where $U$ is the sound velocity in the water (namely about $1500 \mathrm{~m} / \mathrm{sec}$.), consequently $z$ in second. If $z=z_{0}$ at the bottom, $H=U z_{0} . \quad \zeta, \eta$ and $\phi$ are the elevation of the sea surface, elevative velocity of the bottom and the velocity potential in the water, respectively. We adopt the virtual viscosity coefficient $\mu$ for the advantage in the calculation. Then the wave equation is as follows, in which the gravitational effect in the large scale phenomenon appears as the last term in the left hand.

$$
U^{2}\left\{\frac{1}{r} \frac{\partial}{\partial r}\left(r \frac{\partial \phi}{\partial r}\right)+\frac{1}{r^{2}} \frac{\partial^{2} \phi}{\partial \theta^{2}}\right\}+\frac{\partial^{2} \phi}{\partial z^{2}}+\frac{g}{U} \frac{\partial \phi}{\partial 2}=\frac{\partial^{2} \phi}{\partial t^{2}}+2 \mu \frac{\partial \phi}{\partial t}
$$

here, $r$ and $\theta$ are the cylindrical coordinates.

Outside the upper and lower boundaries, we think airless and rigid respectively. Therefore, the two boundary conditions are given as

$$
\frac{\partial^{2} \phi}{\partial t^{2}}+2 \mu \frac{\partial \phi}{\partial t}=\frac{g}{U} \frac{\partial \phi}{\partial z} \quad z=0
$$

and

$$
\frac{1}{U} \frac{\partial \phi}{\partial z}=f(r) \cos n \theta \cdot \chi(\tau)=\eta \quad z=z_{0}
$$




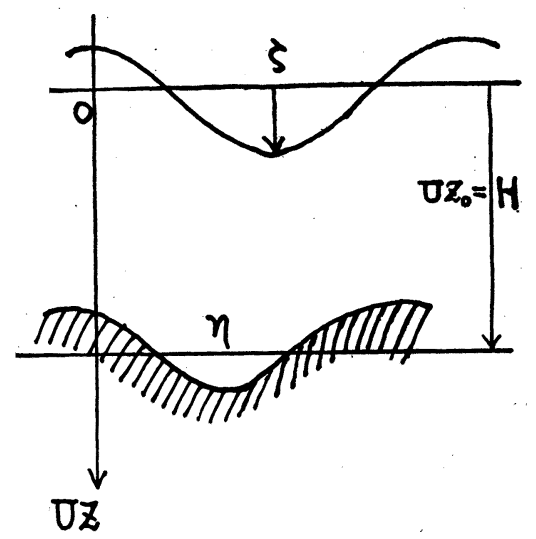

Fig. 2

The solution of (1) is

$$
\phi=\cos n \theta \int_{-\infty}^{\infty} e^{i \alpha_{t}} d \alpha \int_{0}^{\infty}\left(A e^{p_{1} z}+B e^{p_{2} z}\right) J_{n}(k r) d k
$$

here

$$
p_{1,2}=\frac{-g \pm \sqrt{g^{2}-4 U^{2}\left(\alpha^{2}-2 \mu \alpha i-U^{2} k^{2}\right)}}{2 U}
$$

Considering the boundary conditions (2) and (3), (4) becomes

$$
\begin{aligned}
\phi= & \frac{\cos n \theta}{2 \pi} \int_{-\infty}^{\infty} d \alpha \int_{-\infty}^{\infty} \chi(\tau) e^{i \alpha(t-\tau)} \cdot \\
& U \frac{\left\{\left(-\alpha^{2}+2 \mu \alpha i-g / U \cdot p_{1}\right) e^{p_{2} z}-\left(-\alpha^{2}+2 \mu \alpha i-g / U \cdot p_{2}\right) e^{p_{1} z}\right\} d \tau}{\left\{\left(-\alpha^{2}+2 \mu \alpha i-g / U \cdot p_{1}\right) p_{2} e^{p_{2} z_{0}}-\left(-\alpha^{2}+2 \mu d i-g / U \cdot p_{2}\right) p_{1} e^{p_{12} z_{0}}\right\}} \\
& \times \int_{0}^{\infty} J_{n}(k r) k d k \int_{0}^{\infty} J_{n}(k \lambda) f(\lambda) \lambda d \lambda
\end{aligned}
$$

Noting that the surface elevation is given as

$$
\zeta=\frac{1}{g}\left[\frac{\partial \phi}{\partial t}+2 \mu \phi\right]_{\mathrm{z}=0,}
$$

we can calculate $1 / g \cdot\left(\frac{\partial \phi}{\partial t}+2 \mu \phi\right)$ for given $z$, and put $z=0$.

On integrating (6), we assume the poles on the $\alpha$-plane to be represented: as $\alpha_{0}+i \varepsilon$, where $\varepsilon$ and $\mu$ (viscosity coefficient) are small enough. Next, we define a non-dimensional small quantity $\delta$ as follows,

$$
\delta=1 / 2 \cdot\left(\frac{C}{U}\right)^{2}=\frac{g z_{0}}{2 U} \fallingdotseq \frac{H^{\prime}}{450} \ll 1 .
$$

here, $C$ is the velocity of the long wave and the $H^{\prime}$ the depth of the sea in. kilometers. 
H. Miyoshi : Generation of the Tsunami in Compressible Water (Part I)

Now we assume the bottom deformation to be given by the following equations :

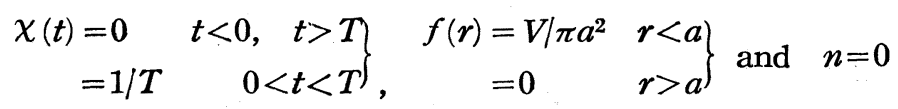

This combination means that the circular area of the bottom, the radius of which is $a$, is elevated uniformly at a constant velocity during the time interval $0<t<T$, the volume of the bottom deformation amounting to $V$.

If we put $m=k a$ and $h=H / a$, and solve the equntions

$$
\delta \tanh X_{0}=\frac{z_{0}^{2} \gamma_{0}^{2} X_{0}}{\left(2 m^{2} h^{2}-z_{0}^{2} \gamma_{0}^{2}\right)}
$$

and

$$
\delta \tan X_{n}=\frac{z_{0}^{2} \gamma_{n}^{2} X_{n}}{\left(2 m^{2} h^{2}-z_{0}^{2} \gamma_{n}^{2}\right)} \quad n=1,2,3, \cdots \quad(10 \mathrm{~b})
$$

with the aids of the parameters $X_{0}$ and $X_{n}$, which are given by

$$
X_{0}^{2}=\delta^{2}+m^{2} h^{2}-z_{0}^{2} \gamma_{0}^{2}
$$

and

$$
X_{n}^{2}=-\delta^{2}-m^{2} h^{2}+z_{0}^{2} \gamma_{n}^{2},
$$

the poles on the $\alpha$-plane are given by

$$
\pm \gamma_{0}+i \varepsilon
$$

and

$$
\pm \gamma_{n}+i \varepsilon
$$

here, it is easily verified that $\lim _{\mu \rightarrow 0} \varepsilon / \mu=1$.

Among the roots of (10a) and (10b), we cast away those corresponding to $X_{0}=$ $X_{n}=0$. Then the integrand takes an indeterminate form.

$$
\begin{aligned}
& \text { Considering (10a, b), } \mu \rightarrow 0 \text { and } \int_{-\infty}^{\infty} d \tau \\
& =\int_{-\infty}^{t} d \tau \text {, we obtain } \\
& \lim _{\mu \rightarrow 0} 1 / g \cdot\left[\frac{\partial \phi}{\partial t}+2 \mu \phi\right] \\
& \begin{array}{ll}
=1 / T \cdot[\xi(t, z)-\xi(t-T, z)] & t>T \\
=1 / T \cdot[\xi(t, z)-\xi(0, z)] & t<T .
\end{array}
\end{aligned}
$$

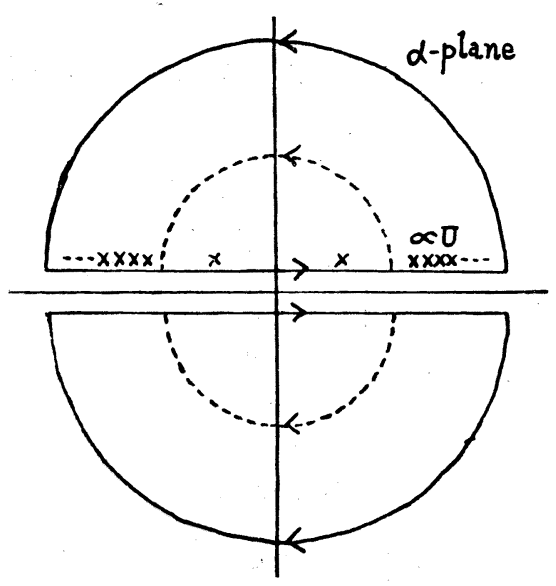

Fig. 3

Semi-circles by full lines: for compressible case, semi-circles by dotted lines; for incompressible case,

upper semi-circles: for $t>\tau$, and lower semi-circles: for $t<\tau$.

where, $\xi(t, z)=V / \pi a^{2} \cdot \frac{z_{0}^{2}}{\delta^{2}} e^{\delta\left(1-z / z_{0}\right)}$ 
$\times\left[\int_{0}^{\infty} \frac{\left\{2 \delta X_{0} \cosh \left(X_{0} z / z_{0}\right)+\left(2 \delta^{2}-z_{0}^{2} \gamma_{0}^{2}\right) \sinh \left(X_{0} z / z_{0}\right)\right\} \gamma_{0} X_{0}^{2} \sin \gamma_{0} t \cdot J_{0}(m r / a) J_{1}(m) d m}{\sinh X_{0}\left(4 m^{2} h^{2} X_{0}^{2}-2 z_{0}^{2} \gamma_{0}^{2} m^{2} h^{2}+z_{0}^{4} \gamma_{0}^{4}+z_{0}^{4} \gamma_{0}^{4} X_{0}^{2} / \delta \sinh ^{2} X_{0}\right)}\right.$

$\left.+\int_{0}^{\infty} \sum_{n=1}^{\infty} \frac{\left\{2 \delta X_{n} \cos \left(X_{n:} z / z_{0}\right)+\left(2 \delta^{2}-z_{0}^{2} \gamma_{n}^{2}\right) \sin \left(X_{n} z / z_{0}\right)\right\} \gamma_{n} X_{n}^{2} \sin \gamma_{n} t \cdot J_{0}(m r / a) J_{1}(m) d m}{\sin X_{n}\left(4 m^{2} h^{2} X_{n}{ }^{2}+2 z_{0}^{2} \gamma_{n}^{2} m^{2} h^{2}-z_{0}^{4} \gamma_{n}^{4}-z_{0}^{4} \gamma_{n}^{4} X_{n}^{2} / \delta \sin ^{2} X_{n}\right)}\right]$

Then the surface elevation is given as

$$
\left.\begin{array}{rlrl}
\zeta & =\frac{1}{T}[\xi(t, 0)-\xi(t-T, 0)] & t>T \\
& =\frac{1}{T}[\xi(t, 0)-\xi(0,0)] & t<T
\end{array}\right\} .
$$

where, writing $\xi(t, 0)$ as $\xi$,

$$
\begin{gathered}
\boldsymbol{e}^{-\delta} \cdot \frac{\pi a^{2}}{V} \xi=2 z_{0}^{2} \int_{0}^{\infty} \frac{\gamma_{0} X_{0} \sinh X_{0} \sin \gamma_{0} t \cdot J_{0}(m r / a) J_{1}(m) d m}{\left\{z^{4} \gamma_{0}^{4}+z_{0}^{4} \gamma_{0}^{4}\left(\sinh ^{2} X_{0} / X_{0}^{2}\right) \delta+4 m^{2} h^{2} \delta \sinh ^{2} X_{0}-2 z_{0}^{2} \gamma_{0}^{2} m^{2} h^{2} \delta\left(\sinh ^{2} X_{0} / X_{0}^{2}\right)\right\}} \\
-2 z_{0}^{2} \int_{0}^{\infty} \sum_{n=1}^{\infty} \frac{\gamma_{n} X_{n} \sin X_{n} \sin \gamma_{n} t \cdot J_{0}(m r / a) J_{1}(m) d m}{\left\{z_{0}^{4} \gamma_{n}{ }^{4}+z_{0}^{4} \gamma_{n}^{4}\left(\sin ^{2} X_{n} / X_{n}{ }^{2}\right) \delta-4 m^{2} h^{2} \delta \sin ^{2} X_{n}-2 z_{0}^{2} \gamma_{n}{ }^{2} m^{2} h^{2} \delta\left(\sin ^{2} X_{n} / X_{n}^{2}\right)\right\}}
\end{gathered}
$$

Then we must search for the values of $X_{0}$ and $X_{n}$ from $(10 \mathrm{a}, \mathrm{b})$ and $(11 \mathrm{a}, \mathrm{b})$.

At first, if we put $X_{0} \fallingdotseq m h$ in (11a), $\gamma_{0}$ becomes very small and (10a) is fulfilled approximately. Next, if we put $X_{n} \fallingdotseq \frac{(2 n-1) \pi}{2}$ in (11b), $\delta \tan X_{n}$ escapes from being very small, and $(10 \mathrm{~b})$ can be established. Putting $b_{n}=\frac{(2 n-1) \pi}{2 h}$, we will expand $X_{0}$ and $X_{n}$ as power series of $\delta$ as follows,

$$
\begin{aligned}
& X_{0}=m h-\delta \tanh m h+\delta^{2} \operatorname{sech}^{2} m h(\tanh m h+1 / 2 m h)+\sim \\
& X_{n}=b_{n} h-\delta \frac{2}{(2 n-1) \pi} \frac{\left(m^{2}-b_{n}^{2}\right)}{\left(m^{2}+b_{n}^{2}\right)}+\sim .
\end{aligned}
$$

Substituting these values in (15), and expanding

$$
\begin{aligned}
\frac{\pi a^{2}}{V} \xi & =(1+2 \delta) \int_{0}^{\infty} \frac{\sin \gamma_{0} t \cdot J_{0}(m r / a) J_{1}(m) d m}{\sqrt{\left(2 \delta / z_{0}^{2}\right) m h \tanh m h \cdot \cosh m h}} \\
& -\frac{3}{2} \delta \int_{0}^{\infty} \frac{\sin \gamma_{0} t \cdot J_{0}(m r / a) J_{1}(m) d m}{\sqrt{\left(2 \delta / z_{0}^{2}\right) m h \tanh m h \cdot \cosh ^{3} m h}}+\sim \\
& +\frac{\pi(1+\delta)}{h^{3}} z_{0} \int_{0}^{\infty} \sum_{n=1}^{\infty} \frac{(-1)^{n}(2 n-1) \sin \gamma_{n} t \cdot J_{0}(m r / a) J_{1}(m) d m}{\sqrt{\left(m^{2}+b_{n}^{2}\right)^{3}}} \\
& -\frac{\delta \pi z_{0}}{h^{5}} \int_{0}^{\infty} \sum_{n=1}^{\infty} \frac{(-1)^{n}(2 n-1)\left(3 b_{n}^{2}-7 m^{2}\right) \sin \gamma_{n} t \cdot J_{0}(m r / a) J_{1}(m) d m}{\sqrt{\left(m^{2}+b_{n}^{2}\right)^{7}}}+\sim
\end{aligned}
$$

where

and

$$
\gamma_{0}=\gamma_{00}\left(1-\delta / 2 \cdot \sec h^{2} m h+\sim\right), \quad \gamma_{00}=\sqrt{\frac{2 \delta}{{z_{0}}^{2}} m h \tanh m h}
$$

$$
\gamma_{n_{0}}=\gamma_{n 0}\left\{1-\delta \frac{\left(m^{2}-b_{n}^{2}\right)}{\left(m^{2}+b_{n}^{2}\right)}+\sim\right\}, \quad \gamma_{n 0}=\frac{h}{z_{0}} \sqrt{\left(m^{2}+b_{n}^{2}\right)} .
$$


In the right-hand side of (17), the first half represents the tsunami and the latter half, the oscillation. (If $U \rightarrow \infty, z_{0} \rightarrow 0(1 / U), \delta \rightarrow 0\left(1 / U^{2}\right)$ and $\frac{2 \delta}{z_{0}^{2}} \equiv g / H$, the former coincides with the solution of the incompressible water, and the latter disappears.) We will designate them $\xi_{r}$ and $\xi_{0}$ respectively.

The former resembles closely to that of the tsunami in the incompressible water as follows :

(1) When $h \gg 1$ (narrow deformation), $\xi_{T}$ coincides with the equation of the tsunami in the incompressible case, the surface elevation being magnified $(1+2 \delta)$-times.

(2) When $h \ll 1$ (broad deformation), $\xi_{T}$ coincides with the incompressible case, the surface elevation being magnified $(1+\delta / 2)$-times, and the time scale is enlarged $(1+\delta / 2)$-times. The latter is proper, for the velocity of the long wave in the compressible liquid decreases $(1-\delta / 2)$-times. In this case, only a ring of elevation followed by another of depression spreads out by the velocity $\sqrt{g H(1-\delta)}$.

(3) When $h \approx 1$ (moderate deformation), the tsunami behaves as (1) in its rear and as (2) in its front. In the middle part, it differs slightly from (1) or (2).

Therefore we shall confine ourselves for the investigation of the latter half of (17), the oscillation by the waves of expansion. Neglecting the terms of orders $\delta, \delta^{2}, \ldots \ldots$, we may write

$$
\frac{\pi a^{2}}{V} \xi_{0}=\frac{\pi}{z_{0}^{2}} \int_{0}^{\infty} \sum_{n=1}^{\infty} \frac{(-1)^{n}(2 n-1) \sin \gamma_{n 0} t \cdot J_{0}(m r / a) J_{1}(m) d m}{\gamma_{n_{0}}{ }^{3}}
$$

where,

$$
\mathrm{t}=\left\{1-\frac{\left(m^{2}-b_{n}^{2}\right)}{\left(m^{2}+b_{n}^{2}\right)} \delta\right\} t
$$

\section{$\S 3$. The Oscillation Immediately after a Broad Deformation}

Now we have $\mathrm{t}=(1+\delta) t$ and $\gamma_{n_{0}}=\frac{h}{z_{0}}\left(b_{n}+\frac{m^{2}}{2 b_{n}}+\sim\right)$. Next, if we put $h(1-$ $\delta / 2)=h^{\prime}$, we obtain

$$
\begin{gathered}
\frac{\pi a^{2}}{V} \xi_{0} \fallingdotseq \frac{8 z_{0}}{\pi^{2}} \sum_{n=1}^{\infty} \frac{(-1)^{n}}{(2 n-1)^{2}}\left\{\sin \frac{(2 n-1) \pi}{2 z_{0}} \mathrm{t} \int_{0}^{\infty} \cos \frac{m^{2} h^{\prime 2} \mathrm{t}}{(2 n-1) \pi z_{0}} \cdot J_{0}(m r / a) J_{1}(m) d m\right. \\
\left.+\cos \frac{(2 n-1) \pi}{2 z_{0}} \mathrm{t} \int_{0}^{\infty} \sin \frac{m^{2} h^{\prime 2} \mathrm{t}}{(2 n-1) \pi z_{0}} \cdot J_{0}(m r / a) J_{1}(m) d m\right\} \\
(7)
\end{gathered}
$$


When $\mathrm{t} \ll \frac{(2 n-1) \pi z_{0}}{m^{2} h^{\prime 2}}$, (21) becomes

$$
\frac{\pi a^{2}}{V} \xi_{0} \fallingdotseq \frac{8 z_{0}}{\pi^{2}} \sum_{n=1}^{\infty} \frac{(-1)^{n} \sin \frac{(2 n-1) \dot{\pi}_{\mathrm{t}}}{2 z_{0}}}{(2 n-1)^{2}} \int_{0}^{\infty} J_{0}(m r / a) J_{1}(m) d m
$$

If we put

$$
\frac{4}{\pi^{2}}\left(\frac{\sin x}{1^{2}}-\frac{\sin 3 x}{3^{2}}+\frac{\sin 5 x}{5^{2}}-\sim\right)=F(x)
$$

(22) further becomes

$$
\left.\begin{array}{rlr}
\frac{\pi a^{2}}{V} \xi_{0} \fallingdotseq-2 z_{0} F\left(\frac{\pi \mathrm{t}}{2 z_{0}}\right) & r<a \\
& \fallingdotseq 0 & r>a
\end{array}\right\}
$$

As the period of $F\left(\frac{\pi^{\mathrm{t}}}{2 z_{0}}\right)$ is $4 z_{0}$, we may put $T=4 z_{0} N$ and $N=N_{0}+\triangle$. Here, $N_{0}$ is an integer and $0 \leqq \Delta<1$. Then, from (14) and (24),

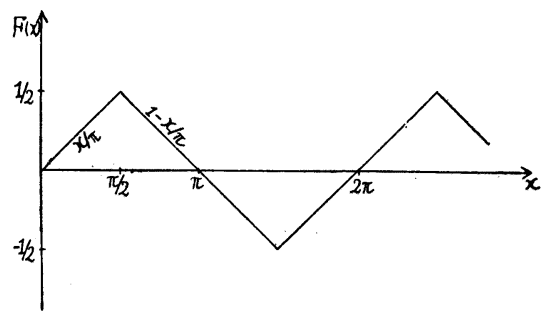

Fig. 4

$$
\begin{array}{rlrl}
\zeta_{0} & =-\frac{2 z_{0}}{T}\left(\frac{V}{\pi a^{2}}\right)\left[F\left(\frac{\pi \mathrm{t}}{2 z_{0}}\right)-F\left(\frac{\pi \mathrm{t}}{2 z_{0}}-2 \pi \Delta\right)\right] & & \mathrm{t}>T(1+\delta) \\
& =-\frac{2 z_{0}}{T}\left(\frac{V}{\pi a^{2}}\right) F\left(\frac{\pi \mathrm{t}}{2 z_{0}}\right) & \mathrm{t}<T(1+\delta)
\end{array}
$$

From (25a), if $\triangle=0$, the oscillation stops as the bottom deformation ceases. But, if $\Delta=1 / 2$, the oscillation is magnified two times. The surface elevationtime diagrams for various $N_{0}$ and $\Delta$ are drawn in Fig. 5 and Fig. 6. The dotted lines represent $\zeta_{T}$ or the elevations in the incompressible cases.

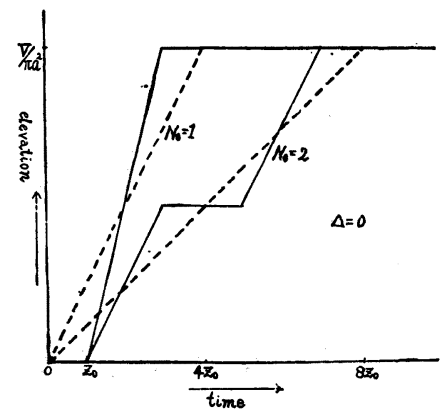

Fig. 5

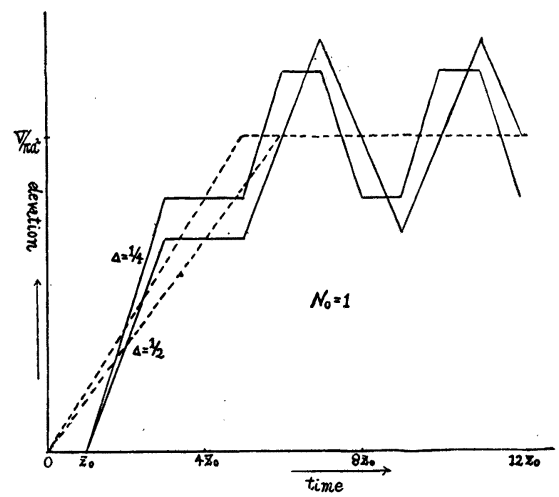

Fig. 6

Acknowledgement. The author expresses his best thanks to Professor $\mathrm{K}$. Hidaka and Professor R. Takahasi for their encouragement. 


\section{References}

Ergin, K. (1952): "Energy Ratio of the Seismic Waves Reflected and Refracted at a Rock-Water Boundary," Bull. Seism. Soc. Amer. 42 (4), p.p. 349-372.

Ewing, M., Worzel, J. L. Hersey, J. B., Press, F. and Hamilton, G. R. (1950) : "Seismic Refraction Measurements in the Atlantic Ocean Basin, Part I," Bull. Seism. Soc. Amer. 40 (3), p.p. 233-242.

Heck, N. H. (1947) : "List of seismic sea waves," Bull. Seism. Soc. Amer. 37 (4), p.p. 269 $-286$.

Imamura, A. (1934): (In Japanese).

Lamb, H. (1932): Hydrodynamics.

Miyabe, N. (1934): "An Investigation of the Sanriku Tsunami Based on Mareogram Data," Bull. Earthq. Res. Inst., Supplementary Volume 1, Part 1, p.p. 112-126.

Pidduck, F. B. (1912) : "The Wave-Problem of Cauchy and Poisson for finite Depth and slightly Compressible Fluid," Proc. Roy. Soc. Lond. Vol. 86, p.p. 396-

Sverdrup, H. U., et al, (1946) : “The Oceans."

Takahasi, R. (1942): (In Japanese).

Takahasi, R. (1951) : "An Estimate of Future Tsunami Damage Along the Pacific Coast of Japan,” Bull. Earthq. Res. Inst., Vol. 29, Part I, p.p. 71-95. 